\title{
DESCRIPTION OF THE ADSORPTION EQUILIBRIUM WITH CONSIDERATION TO THE DECREASING AVAILABILITY OF SPACE IN THE COURSE OF MOBILE ADSORPTION OF A SINGLE GAS ON A HOMOGENOUS SURFACE OF SOLID
}

\author{
Jerzy K. Garbacz ${ }^{1)}$, Ewa Kopkowska ${ }^{2)}$, Barbara Rymian ${ }^{3)}$ \\ 1) University of Technology and Life Sciences in Bydgoszcz, Faculty of Environmental Engineering and Environmental Physical Chemistry, Poland \\ 2) University of Nicolaus Copernicus in Toruń Collegium Medicum in Bydgoszcz, Faculty and Institute of Bio-Chemistry, Poland \\ ${ }^{3)}$ University of Nicolaus Copernicus in Toruń Collegium Medicum in Bydgoszcz, Faculty and Institute of Hygiene and Epidemiology, Poland
}

\section{ABSTRACT}

The subsequent stages of the process of formulation of the equation for gas adsorption on a homogenous surface of a solid adsorbent were presented based on the general expression for the canonical ensemble of the mobile single-component adsorption monolayer. The method of formulating the configuration integral of the proposed model was discussed in detail where the role both of the attraction and repulsion between adsorbed molecules was emphasised. The expression for the probability of finding a molecule in a specified point on a surface of an adsorbent was modified by determining its magnitude by the adsorbent concentration. The expression for the so-called effective surface of the adsorbent was obtained by adapting a two-dimensional analogue equation of state hard spheres - Van der Waals equation (2D-vdW) and Reis-Frisch-Lebowitz equation accordingly (2D-RFL). As a result, two new adsorption equations were formulated which differ in detail concerning the adsorbate-adsorbate repulsion. On each of these equations theoretical analysis was performed in terms of two-dimensional phase transformation. In both cases it was proved that the proposed solution allows for the presence of two-phase transformations of the first type which is the gas-liquid condensation and solidification liquid-solid. The verification of the given approach was supplemented by the description of the experimental data given in reference literature and by obtaining a very good correlation between the theory and experiment.

Keywords: adsorption, mobility, isotherm

ARTICLE INFO

PolHypRes 2018 Vol. 64 Issue 3 pp. 25 - 38

ISSN: 1734-7009 elSSN: 2084-0535

DOI: $10.2478 /$ phr-2018-0016

Pages: 13 , figures: 4 , tables: 3

page www of the periodical: www.phr.net.pl

Publisher

Polish Hyperbaric Medicine and Technology Society
Original article

Submission date: $15.06 .2018 \mathrm{r}$.

Acceptance for print: 25.07.2018 r. 


\section{INTRODUCTION}

In numerous discussions on intermolecular interactions in the adsorption layer, the main emphasis was placed on mutual attraction of adsorbed molecules [1-5]. In the mentioned publications the influence of these interactions on such adsorption characteristics as isotherm, heat (differential and integral) and thermal capacity of the adsorption layer was analysed. The interactions themselves were divided into non-specific, i.e. dispersive (van der Waals type) and specific, i.e. electrostatic (e.g. dipole-dipole) or associative (quasichemical) interactions.

The valuable results of these analyses are an indisputable indication of the validity of the assumed research direction and a high assessment of its cognitive potential. In the opinion of the authors of this article, there is, however, a fundamental concern that the unintended result of such an intense focus on one type of interactions may be an underestimation of the other, i.e. the adsorbate-adsorbate repulsion. Although repulsion is not omitted in theoretical approaches (as results from the assumption of a finite volume of own admolecules), it seems to be incomplete and, what is more important, possible to consider more fully. The aim of this paper is to take up this challenge with the use of statistical thermodynamics formalism.

\section{DERIVATION OF ADSORPTION EQUATIONS}

Let us consider a single-component fully mobile monolayer physically adsorbed on a homogeneous surface (in terms of energy) of a solid adsorbent. Let us assume that this layer (adsorbate) remains in thermodynamic equilibrium with the non-adsorbed isocomponent gas (adsorbent), whereas the surface of the adsorbent is only a source of an external potential responsible for the adsorption phenomenon. The twodimensional character of the monolayer, in the model sense, allows to present its canonical sum of state, $Z_{a}$, as the following general expression [6]:

$$
Z_{a}(T, A)=\frac{1}{N ! h^{2 N}} \int d \boldsymbol{r}^{N} d \boldsymbol{p}^{N} \exp \left[-\frac{H\left(\boldsymbol{r}^{N}, \boldsymbol{p}^{N}\right)}{k T}\right]
$$

where $\mathrm{N}, \mathrm{T}, \mathrm{A}$ and $\mathrm{H}$ denote the number of adsorbate molecules, temperature, surface area of the adsorbent and the total energy of the adsorptive layer, respectively, whereas $r^{N}$ and $p^{N}$ are $2 \mathrm{~N}$ - dimensional vectors, in the order, positions and momentums of all adsorbate molecules. Physical constants, i.e. Planck's constants and Boltzmann's constants, are customarily marked with symbols $h$ and $k$. It is worth emphasizing that the preintegral factor of equation (1) takes into account two quantum effects, not included in the classic sum of states, i.e. indistinguishability of identical molecules $(N !)$ and the uncertainty of angular momentum $\left(h^{2 N}\right)$.

The division of total energy, $H\left(r^{N}, p^{N}\right)$, into parts: translational, $T\left(p^{N}\right)$ and configurational, $V\left(r^{N}\right)$ along with presenting the kinetic energy of a molecule with the mass $m$ as $p^{2} / 2 m$, and then integration will give: $Z=\frac{t^{-2 N}}{N !} f_{a}^{N} \int d \boldsymbol{r}^{N} \exp \left[-\frac{V\left(\boldsymbol{r}^{N}\right)}{k T}\right]$,

where $f_{a}$ is the sum of the internal states of the degrees of freedom of the adsorbate molecule, whereas:

$$
t=\frac{h}{\sqrt{2 \pi m k T}}
$$

In order to simplify the configuration integral $Q$ of equation (2), let's use the theorem of the mean value, known from the integral calculus. In concord with this theorem:

$Q=A_{e f}^{N}\left\langle\exp \left(-\frac{V}{k T}\right)\right\rangle$

Above $A_{e f}$ is the effective surface area of the adsorbent (for the non-ideal layer $A_{e f} \leq A$ ), while the symbol $\langle\cdots\rangle$ indicates the average value of the expression contained in parentheses. Without limiting the range of potential energy variability, $V$, we can write down the following:

$\left\langle\exp \left(-\frac{V}{k T}\right)\right\rangle=\int_{-\infty}^{+\infty} \rho(V) \exp \left(-\frac{V}{k T}\right) d V$,

where $\rho(V) d V$ is the probability that the potential energy of the adsorption layer is in the range $(V, V+d V)$.

In a canonical ensemble [7]:

$\rho(V)=\frac{g(V) \exp \left(-\frac{V}{k T}\right)}{Q}$,

where $g(V)$ is the density of the configurational states within the range of potential energy $(V, V+d V)$.

Bilateral logarithming of equation (6) and then developing the right side of the equation into a power series around the mean value of the argument, $\bar{V}$, gives:

$\ln g(V)=\ln g(\bar{V})+\left\{\left[\frac{\partial \ln g(V)}{\partial V}\right]_{V=\bar{V}}-\frac{1}{k T}\right\}(V-\bar{V})+$
$\frac{1}{2}\left[\frac{\partial^{2} \ln g(V)}{\partial V^{2}}\right]_{V=\bar{V}}(V-\bar{V})^{2}+\cdots$

The first derivative of the function $\ln g(V)$ has a known thermodynamic interpretation [7] and for $V=\bar{V}$ is equal to $1 / \mathrm{kT}$. This means that in equation (7) the segment proportional to the first power of the differential $(V-\bar{V})$ disappears. Neglecting the third and higher powers of this differential we can write down the following:

$\rho(V)=\rho(\bar{V}) \exp \left[-\frac{(V-\bar{V})^{2}}{2 k T^{2} C_{A}}\right]$,

where $C_{A}=\left(\frac{\partial V}{\partial T}\right)_{A} C_{-} \mathrm{A}=(\partial \mathrm{V} / \partial \mathrm{T})_{-} \mathrm{A}$ is the configurational part of the thermal capacity of the adsorption layer (with a constant adsorbent surface, $\mathrm{A}$ ).

The form of the factor $\rho(\bar{V})$ is obtained by taking into account the condition of normalisation of the total probability to unity. The appropriate integration (within $\pm \infty$ ) gives:

$\rho(\bar{V})=\frac{1}{\sqrt{2 \pi k T^{2} C_{A}}}$

At this point it should be noted that $V, \bar{V}$ and $\mathrm{C}_{A}$ are extensive values and can be presented as, $N v, N \bar{v}$ i $N C_{A}$ respectively, where the intensive variables $v, \bar{v}$ and $c_{A}$ are their molecular values. In this situation, from the combination of equations (8) and (9), we obtain: 
$\rho(V) d V=\frac{1}{\sqrt{2 \pi k T^{2} c_{A} / N}} \exp \left[-\frac{(v-\bar{v})^{2}}{2 \pi k T^{2} c_{A} / N}\right] d v$,

where the expression $k T^{2} c_{A} / N$ is a dispersion, $\sigma^{2}$, of the Gaussian distribution, $\rho(v)$. This value moves within the thermodynamic range of $N \rightarrow \infty$ towards zero, which means that the distribution $\rho(v)$ itself passes into the Dirac delta-function $\delta(v-\bar{v})$. Therefore, returning to equation (5), we may conclude that for the macroscopic system, which is certainly the adsorption layer we will obtain the following:

$\left\langle\exp \left(-\frac{V}{k T}\right)\right\rangle \frac{N \rightarrow \infty}{\sigma^{2} \rightarrow 0} \exp \left(-\frac{\bar{V}}{k T}\right)$

Further progress in these considerations requires findings regarding the form of the effective surface area of the adsorbent, Aef and the mean potential energy of the adsorbate, $\bar{V}$. As for the first one, let us notice that the two-dimensional pressure, $\varphi$, of a gas whose molecules do not attract each other is expressed by the following relation: $\varphi A_{f}=N k T$, where $A_{f}$ means the part of the total adsorbent area available for adsorbed molecules (due to non-zero molecule's own volume, $A_{f} \leq$ $A$ ). On the other hand, the same equation of state (and thus the same form of $A_{f}$ )) results from a known thermodynamic dependence: $\varphi=k T(\partial \ln Z / \partial A)$ with determined $\mathrm{T}$ and $\mathrm{N}$ [7]. However, because in the sum of states (2), in the absence of an adsorbate-adsorbate attraction, the dependence on A concerns only the effective area, $A_{\text {ef, }}$ therefore, after a simple transformation:

$\ln A_{e f}=\int A_{f}^{-1} d A+C$,

where $\mathrm{C}$ is the integration constant, the proper choice of which must meet the condition: $A_{e f}(N=0)=A$.

The usefulness of the equation (12) will be illustrated by two examples, which in the latter part of this article will be used to formulate an appropriate adsorption equation. For this purpose, we will apply twodimensional analogues of van der Waals (2D-vdW) and Reis-Frisch-Lebowitz (2D-RFL) models, for which $A_{f}$ is respectively equal to [5]:

fog 2D-vdW:

$A_{f}=A\left(1-\frac{N b_{0}}{A}\right)$

And for 2D-RFL:

$A_{f}=A\left(1-\frac{N b_{0}}{2 A}\right)^{2}$

Both discussed models, in their threedimensional variants, treat the molecule as a rigid sphere. The projection on the adsorbent surface imitates its large circle, hence the common reference to a hard discs model [8] in the case of a two-dimensional variant. In equations (13) and (14) the parameter $b_{0}$ is the second virial coefficient of the fluid of rigid discs, and its value equals double the surface of the molecule's own large circle a rigid sphere. Subjecting dependencies (13) and (14) to transformation (12) leads to the following expressions defining the effective area of the adsorbent, $A_{f}$ : for 2D-vdW:

$A_{\text {ef }}=A\left(1-\frac{N b_{0}}{A}\right)$

and for 2D-RFL:

$A_{e f}=A\left(1-\frac{N b_{0}}{2 A}\right) \exp \left(-\frac{N b_{0} / 2 A}{1-N b_{0} / 2 A}\right)$

At this point it should be stressed that the identicality of the right sides of equations (13) and (15) constitutes a special case and not a rule, as evidenced by the difference between equations (14) and (16). Let us now move on to the formulation of an appropriate expression for mean potential energy, $\bar{V}$. We should bear in mind that in the considerations on adsorption, this value is traditionally divided between two types of interactions, i.e. "vertical". (adsorbate-adsorbent) and 'horizontal'. (adsorbate-adsorbate). The sum of both shares may be written in the following order:

$\bar{V}=\bar{V}_{a-s}+\bar{V}_{a-a}$

The component $\bar{V}_{a-s}{ }^{-} \mathrm{V}$ takes into account, inter alia, the vibration energy that the absorbed molecule performs perpendicularly to the surface of the adsorbent. If we assume their harmonisation:

$\bar{V}_{a-s}=\frac{1}{2} N h v+\bar{V}_{a-s}^{*}$,

where $N h v / 2$ is the total zero energy of all admolecules, in which $v$ is the vibration frequency of a single harmonic oscillator.

Usually in adsorption theory formulae the adsorption potential, $E_{0}$, is used instead of energy $\bar{V}_{a-s}$. The relationship between these quantities is expressed as $N E^{0}=-\bar{V}_{a-s}^{*}$, thus, according to the equation (18):

$N E^{0}=-\bar{V}_{a-s}+\frac{1}{2} N h v$

In the classical limit the zero-vibration energy can be taken as the conventional zero potential energy, which simplifies the relation (19) to the form: $\bar{V}_{a-s}=-N E^{0}$.

As for the second component of the right side of equation (17), let us use the assumption of additivity of potential energy to the interacting pairs of molecules. Within this approximation:

$\bar{V}_{a-a}=\frac{N(N-1)}{2} \beta_{1-2} \stackrel{N \rightarrow \infty}{\longrightarrow} \frac{N^{2}}{2} \beta_{1-2}$

where $\beta_{1-2} \beta$ is the average energy of the interaction of a pair of particles conventionally marked as "1" and "2". For continuous energy distribution:

$\beta_{1-2}=\iint d \boldsymbol{r}_{1} d \boldsymbol{r}_{2} u\left(\boldsymbol{r}_{1}, \boldsymbol{r}_{2}\right) \rho\left(\boldsymbol{r}_{1}, \boldsymbol{r}_{2}\right)$

Above, $\boldsymbol{r}_{1}$ and $\boldsymbol{r}_{2}$ are vectors, generally dependent on the position and orientation of the molecules "1" and "2", $u\left(\boldsymbol{r}_{1}, \boldsymbol{r}_{2}\right) \mathrm{u}$ is the potential of mutual interaction between these molecules, while $\rho\left(\boldsymbol{r}_{1}, \boldsymbol{r}_{2}\right)$ is the probability density of the event: molecule "1" at $\boldsymbol{r}_{1}$ point and (simultaneously) molecule "2" at $\boldsymbol{r}_{2}$ point. 
We now have a complete set of dependencies constituting the sum of states of the adsorption layer, $\mathrm{Z}_{\mathrm{a}}$, (equation (1)). This in turn allows us to formulate an expression defining the chemical potential of adsorbate, $\mu_{-}$a, equal, according to the definition [6], to $-k T\left(\partial \ln Z_{a} /\right.$ $\partial N)_{T, A}$.

The final adsorption equation will be obtained using the equilibrium condition between the adsorption layer and the non-adsorbed gas, $\mu_{a}=\mu_{g}$, where $\mu_{g}$ is the chemical potential of the adsorptive. Due to the drastic difference in density between the two phases under consideration, we can assume the perfection of the adsorptive without making a significant error, thus we assume that [ ] $\mu_{g}=k T \ln \left[p t^{3} /\left(k T f_{g}\right)\right]$ where $p$ is the equilibrium pressure of the adsorbent and $f_{g}$ is the internal sum of the states of the non-absorbed molecule.

The result of the respective substitutions and routine transformations are the following adsorption equations:

for 2D-vdW:

$p=\frac{\theta}{K_{H}(1-\theta)} \exp \left[\frac{\theta}{1-\theta}-\frac{2 \alpha_{2}}{k T \omega_{0}} \theta(1-\theta)(1-2 \theta)\right]$

and for 2D-RFL:

$p=\frac{\theta}{K_{H}(1-\theta)} \exp \left[\frac{\theta(3-2 \theta)}{(1-\theta)^{2}}-\frac{2 \alpha_{2}}{k T \omega_{0}} \theta(1-\theta)^{3}(1-3 \theta)\right]$

The $\omega_{0}$ above is the area per molecule of adsorbate in a tightly packed monolayer and is equal to $b_{0}$ for the 2D-vdW model and $b_{0} / 2$ for the 2D-RFL model, respectively. Moreover, $\theta$ is the dimensionless degree of filling up of the adsorbent surface, with values in the range $[0,1]$, determined by the relation: $\theta=N \omega_{0} / A$, while the isothermal constant $K_{H}$, traditionally called Henry's constant, is given by the expression:

$K_{H}=\frac{t \omega_{0}}{k T} \frac{f_{a}}{f_{g}} \exp \left(\frac{E^{0}}{k T}\right)$

The relationships (28) and (29) formulated above constitute the subject of analysis in the aspect of two-dimensional phase transformations of adsorbate, as well as the theoretical basis for the description of experimental adsorption isothermal processes.

\section{Phase transformations in a MOBILE ADSORPTION MONOLAYER}

The model of adsorption allowing the possibility of phase transition in the adsorption layer determines, out of necessity, the coordinates of the point of inflection at the so-called critical isotherm. It is a saddle point at which both the first and second derivative of the adsorption pressure are zeroed in relation to the degree of filling up of the adsorbent surface. It can be easily demonstrated that this condition results in a system of equations:

$\left(\frac{\partial \ln p}{\partial \theta}\right)_{T}=0$

and

$\left(\frac{\partial^{2} \ln p}{\partial \theta^{2}}\right)_{T}=0$
The solutions (if any) of the above set are critical values of the degree of filling, $\theta_{c}$ and the parameter of adsorbate-adsorbate interactions, $\left(2 \alpha_{2} / k T b_{0}\right)_{c}$. Aside from results obtained for the models of localised adsorption, let us recall here the already mentioned equation of Hill-de Boer, which proves to be the lowdensity limit of our equation (28). The function $p(\theta)$ has the form:

$p=\frac{\theta}{K_{H}(1-\theta)} \exp \left(\frac{\theta}{1-\theta}-\frac{2 \alpha_{2}}{k T b_{0}} \theta\right)$

The set of equations (31) with the function $p(\theta)$ represented by the relation (32) has one solution with the coordinates of the critical point: $\theta_{c}=1 / 3$ and $\left(2 \alpha_{2} / k T b_{0}\right)_{c}=27 / 4$ (both values are accurate, therefore we provide them in the fractional form). The physical interpretation adopted in the literature defines this solution, by analogy to three-dimensional transformation, as a critical point of two-dimensional adsorbateadsorbate condensation.

Let us treat the quoted result as a reference to the results of a similar analysis for equations (28) and (29). The use of the first of these relationships as a function of $p(\theta)$ reduces the system of equations (31) to the following forms:

$30 \theta_{c}^{3}-42 \theta_{c}^{2}+15 \theta_{c}-1=0$

and

$\left(\frac{2 \alpha_{2}}{k T b_{0}}\right)_{c}=\frac{1}{\theta_{c}\left(1-\theta_{c}\right)^{2}\left[1-\theta_{c}\left(1-\theta_{c}\right)\right]}$

The physical sense only applies to those solutions of the above system for which, at the same time $0 \leq \theta_{c}<1$ and $\left(2 \alpha_{2} / k T b_{0}\right)_{c}>0$. This means that because of equation (33b), it is necessary to exclude from the range $[0 ; 1)$ a sub-range with ends on both sides $\theta_{c}=1 / 2 \pm \sqrt{3} / 6$ (in a latter part of the paper all approximate numbers are given to the four significant digits), in which one of the three elements of the equation (33) is located, i.e. $\theta_{c}=0.4453$. The remaining elements, together with the corresponding critical values of the adsorbate-adsorbate interaction parameter, form the following pair of solutions to the system of equations (33):

$\theta_{c}=0,08618,\left(2 \alpha_{2} / k T b_{0}\right)_{c}=26,34(34)$

oraz

$\theta_{c}=0,8685,\left(2 \alpha_{2} / k T b_{0}\right)_{c}=211,6$

The existence of two physically acceptable critical points radically differentiates the equation (28) from the Hill-de Boer isotherm, suggesting the possibility of the occurrence of two-phase transitions in the adsorption layer. This possibility seems to be confirmed by calculations made for the sample values, $K_{H}=1$ and $\left(2 \alpha / k T b_{0}\right)=300$ (the former is irrelevant for the shape of the adsorption isotherm, while the latter was to be higher than each of the solutions of the equation (33b)). The results of these calculations show two non-monotonic areas of the function (28).

The first one, corresponding to a low filling of the adsorbent surface, is characterised by a significant 
change in adsorbate density. Replacement of the loop with a straight-line segment determines the "leap" of the value $\theta$ between $2.900 \cdot 10^{-5}$ and 0.4257 , which corresponds to the adsorbate compression coefficient of nearly 14 680. In the second non-monotonic region (28) the change $\theta$ occurs in the range between 0.7855 and 0.9444, which means that the adsorbate density compression is only 1.202 .

The interpretation of the above results in terms of phase transitions leads to the conclusion that they are first type transitions (in both cases there is a phase density change). However, while the former can be seen as two-dimensional gas-liquid condensation, the latter certainly occurs with the participation of two condensed phases and logically corresponds to the two-dimensional liquid-solid solidification. Such a thesis, although cognitively undoubtedly attractive, seems premature with the present premises. It cannot be ruled out upfront that the obtained solution is an artifact resulting from the adoption of a strongly approximate expression (13) to determine the available surface of the adsorbent, $A_{f}$. Let us recall that according to this formula, the most tightly packed molecules of the adsorbate block only 50 per cent of the total area, while for circles of equal diameter arranged in a plane in a strictly hexagonal configuration this degree equals $\pi \sqrt{3} / 6$, or almost 91 per cent (even in a less favourable regular arrangement it is still over 78 per cent).

In this situation, it can be expected that the repetition of the above test using equation (29) as a function of $p(\theta)$ will allow to obtain a more reliable reference to the concept of two-phase transitions in the adsorbent layer. The 2D-RFL model assumes that molecules in a tightly packed monolayer occupy 100 per cent of the total adsorbent area, which is a value much closer to the geometric result and, what is important, unlike the 2D-vdW model, causes an overestimation of this result.

Equations (31a) and (31b) are now equivalent to the set of equations:

$$
\begin{aligned}
& 105 \theta_{c}^{4}+30 \theta_{c}^{3}-100 \theta_{c}^{2}+26 \theta_{c}-1=0 \\
& \left(\frac{2 \alpha_{2}}{k T b_{0}}\right)_{c}=\frac{1+\theta_{c}}{2 \theta_{c}\left(1-\theta_{c}\right)^{5}\left[1-5 \theta_{c}\left(2-3 \theta_{c}\right)\right]}
\end{aligned}
$$

The solutions of the equation (36a) are solely real numbers with values: $-1.229,4.674 \cdot 10^{-2}, 0.2606$ and 0.6364 respectively. The first one is obviously devoid of physical sense, while the third one belongs to the range with the extremities $\theta_{c}=\frac{1}{3} \pm \sqrt{10} / 15$ excluded by equation (36b).

In the end we receive two pairs of solutions, as for the 2D-vdW model, this time with the following values:

$\theta_{c}=4,672 \cdot 10^{-2},\left(2 \alpha_{2} / k T b_{0}\right)_{c}=25,16$

and

$\theta_{c}=0,6364,\left(2 \alpha_{2} / k T b_{0}\right)_{c}=284,5$

Calculations carried out for the same values of $K_{H}$ and $\left(2 \alpha / k T b_{0}\right)$ also confirm the presence of two nonmonotonic areas here (this time for function (29)), and replacing loops with straight segments gives incremental changes in the degree of adsorbent surface filling in the ranges $1.135 \cdot 10^{-5}<\theta<0.2816$ and $0.5933<\theta<$ 0.6816 , which corresponds to adsorbate compression ratios of 23930 and 1.149, respectively. The obtained result, in the qualitative sense identical to the $2 \mathrm{D}$-vdW model, strengthens, according to the conservative opinion of the authors, the argumentation in favour of the theoretical possibility of two-phase transformations in the adsorption layer.

A separate issue, however, is the possibility of an experimental observation of these transformations, especially two-dimensional solidification. This phenomenon, if it occurs, requires extremely high pressure corresponding, as it results from the abovementioned considerations, to a dimensionless product $p K_{H}$ with values not lower than 2,650·1011 according to the 2D-vdW model and 5,335 $10^{10}$ according to the 2D-RFL model. These conditions go beyond the reality of a typical adsorption measurement to such an extent that the discussed phase transition can now only be the subject of theoretical considerations. This does not change the fact, however, that this really interesting aspect indicates the complexity of the consequences of fuller consideration of adsorbate-adsorbate repulsion in the description of adsorption equilibrium, which has thus far been underestimated.

\section{DESCRIPTION OF EXPERIMENTAL DATA}

The usefulness of the equations (28) and (29) derived above was verified by their application to describe experimental isotherms of adsorption. Using the best fit method, it was possible to determine the optimal values of such model parameters as Henry's constant, $\mathrm{K}_{\mathrm{H}}$, dispersion interaction constant, $2 \alpha / k T b_{0}$ and capacity of the adsorption monolayer, $a_{m}$. The last of these parameters occurs, implicite, in the degree of saturation of the surface of the adsorbent, the alternative definition of which has a form: $\theta=a / a_{m}$, where the numerator denotes the value of adsorption under the given finite equilibrium pressure of the adsorptive. Nine experimental data sets taken from the literature [5] and related to the adsorption of single gases on different types of thermally graphitised carbon black were described. Characteristics of the described adsorption systems are presented in Table 1. 
Characteristics of the adsorption systems under theoretical description.

\begin{tabular}{cll}
\hline \multicolumn{2}{c}{ Adsorbate } & $\mathrm{T}(\mathrm{K})$ \\
\hline 1. & nitrogen & 78,2 \\
\hline 2. & benzene & 293,2 \\
\hline 3. & carbon dioxide & 193,2 \\
\hline 4. & ethane & 176,2 \\
\hline 5. & ethene & 176,2 \\
\hline 6. & n-hexane & 293,2 \\
\hline 7. & methane & 113,2 \\
\hline 8. & methylocyclohexane & 293,2 \\
\hline 9. & perfluoromethylocyclohexane & 293,2 \\
\hline
\end{tabular}

Optimal values of model parameters are given in

Tables 2 (equation 28) and 3 (equation 29), supplementing each of these Tables with a column of values of correlation ratio, $r$, adopted as a measure of matching the description with experimental data.

Optimization results for the parameters of equation (28)

\begin{tabular}{lllll}
$\begin{array}{l}\text { Adsorption } \\
\text { system }\end{array}$ & $\mathrm{K}_{\mathrm{H}}$ & $\frac{2 \alpha_{2}}{k_{B} T b_{0}}$ & $\begin{array}{l}\mathrm{am} \\
\left(\mu \mathrm{mol} / \mathrm{m}^{2}\right)\end{array}$ & $\mathrm{r}$ \\
\hline 1. & 135,3 & 57,34 & 20,68 & 0,9940 \\
\hline 2. & 7,734 & 13,48 & 10,46 & 0,9996 \\
\hline 3. & 0,3800 & 16,01 & 31,86 & 0,9979 \\
\hline 4. & 3,826 & 38,21 & 18,55 & 0,9979 \\
\hline 5. & 5,620 & 26,52 & 17,94 & 0,9987 \\
\hline 6. & 74,83 & 20,21 & 6,572 & 0,9935 \\
\hline 7. & 2,717 & 49,86 & 24,14 & 0,9974 \\
\hline 8. & 57,92 & 14,96 & 6,378 & 0,9962 \\
\hline 9. & 105,4 & 41,75 & 4,739 & 0,9944 \\
\hline
\end{tabular}

Optimization results for the parameters of equation (29)

\begin{tabular}{lccll}
$\begin{array}{l}\text { Adsorption } \\
\text { system }\end{array}$ & $\mathrm{K}$ & $\frac{2 \alpha_{2}}{k_{B} T b_{0}}$ & $\begin{array}{l}\mathrm{am} \\
\left(\mu \mathrm{mol} / \mathrm{m}^{2}\right)\end{array}$ & $\mathrm{r}$ \\
\hline 1. & 32,64 & 76,85 & 33,51 & 0,9942 \\
\hline 2. & 4,543 & 14,50 & 17,25 & 0,9996 \\
\hline 3. & 0,2002 & 16,43 & 54,37 & 0,9973 \\
\hline 4. & 4,829 & 28,00 & 28,96 & 0,9982 \\
\hline 5. & 4,077 & 27,22 & 28,27 & 0,9983 \\
\hline 6. & 9,470 & 33,97 & 12,12 & 0,9942 \\
\hline 7. & 2,717 & 49,86 & 24,14 & 0,9974 \\
\hline 8. & 29,44 & 21,58 & 10,43 & 0,9970 \\
\hline 9. & 2,259 & 50,29 & 9,390 & 0,9943 \\
\hline
\end{tabular}

An illustration of such matching for selected examples of adsorption data is shown in the following figures 1-4. 


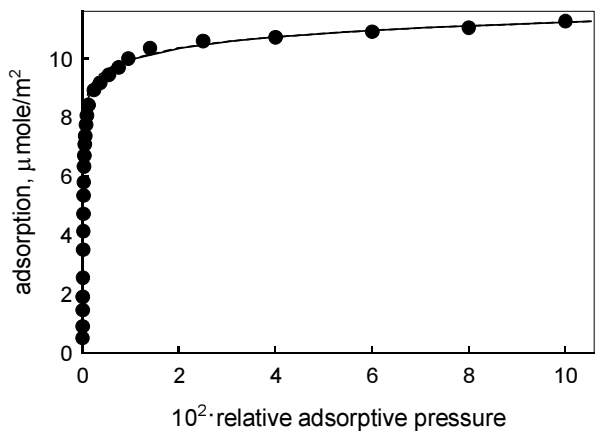

Fig. 1 Adsorption isotherm for the system 1. Points represent experimental data, solid line - acc. to eq. (28), dashed line - acc. to eq. (29).

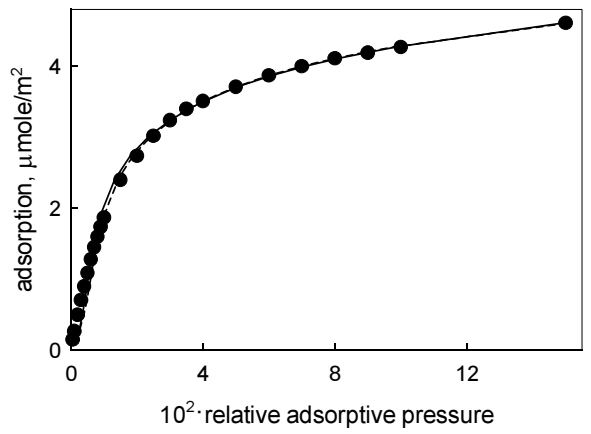

Fig. 2 Adsorption isotherm for the system 2. Points represent experimental data, solid line - acc. to eq. (28), dashed line - acc. to eq. (29).

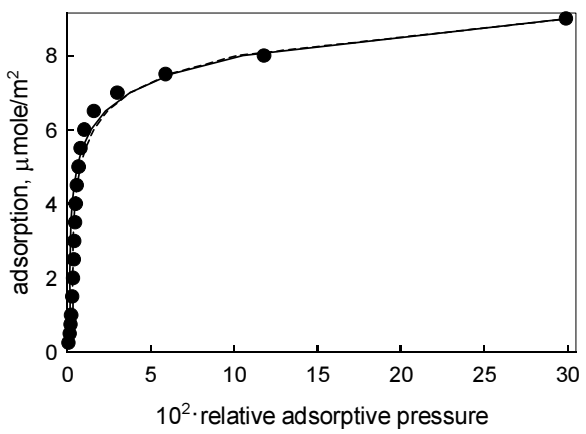

Fig. 3 Adsorption isotherm for the system 4. Points represent experimental data, solid line - acc. to eq. (28), dashed line - acc. to eq. (29).

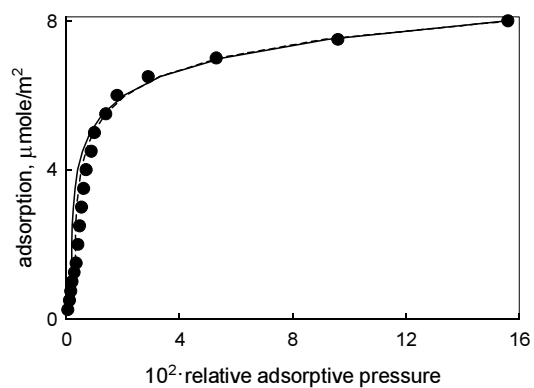

Fig. 4 Adsorption isotherm for the system 5. Points represent experimental data, solid line - acc. to eq. (28), dashed line - acc. to eq. (29). 
Both the analysis of correlation ratio values ( $r>0.99$ for each system) and the presented illustrations where the curves generated by equations (28) and (29) are practically identical proves the high usefulness of the two equations formulated in this work for the interpretation of experimental adsorption data.

\section{Conclusions}

The considerations presented in this paper proved the significant influence of adsorbate-adsorbate interactions on the form and characteristics of the adsorption equation. The main findings are as follows:

1. It was found reasonable to include in the theoretical description of the decreasing character of the probability of availability of adsorption space in the function of adsorbate concentration.

2. The adsorption equations based on the above assumption differ from the previous solutions by a radically different, because non-monotonic, segment of interactions in the adsorption layer.

3 . The most important thermodynamic consequence of the proposed solutions is that each of them envisages not a single, but two two-dimensional phase transitions of the first type.

4. The derived adsorption equations describe experimental adsorption data with very high accuracy.

\section{REFERENCES}

Kiselev A. V., Poshkus D. P., Yashin Ya. I., Molekularnyie Osnovy Adsorbcionnoy Khromatografii, Moscow, izd. Khimiya, 1986, pp. 79-127; Kiselev A. V., Mezhmolekularnyie Vzaimodeysviya v Adsorpcii i Khromatpgrafii, Moscow, izd. Vysshaya Shkola, 1986, pp. 160-221;

Linsen B. G. (ed.) Physical and Chemical Aspects of Adsorbents and Catalysis, London and New York 1960, p.p. 82-125;

Ross S., Olivier J. P., On Physical Adsorption, Willey and Sons, Inc., New York-London_Sydney 1964, p.p. 103-122;

Garbacz J. K., Kopkowski A., Description of adsoprtive balance in the system: a single gaseous komponent - partially mobile adsoptive phase on a homogenous surface, ed. BTN, ISSN 0137-9622, Bydgoszcz 1995, pp. 14-63;

6. Garbacz J. K., Fractal description of the partially mobile single gas adsorption on energetically homogeneous solid adsorbent, Colloid Surf., A143, 1998, pp.95-101;

7. Stecki J. Termodynamika statystyczna, PWN Warszawa 1971, pp.424-452

8. Garbacz J. K., The concept of hard discs in description of association of molecules in non-localized adsorption monolayer on homogeneous surfaces, Polish J. Chem.; 52, 1978, pp 993-1000;

9. Garbacz J. K., Towards more accurate specification in cases of multilayer adsorption on homogeneous surfaces, Polish J. Chem.; 52 , 1978, 1973-1983

prof. dr hab. Jerzy K. Garbacz

Uniwersytet Technologiczno-Przyrodniczy w Bydgoszczy, Wydział Budownictwa, Architektury i Inżynierii Środowiska Katedra Ekoinżynierii i Fizykochemii Środowiska

ul. Sucha 9, 85-789 Bydgoszcz

(52) $3408440 / 3408445$

jerzy.garbacz@utp.edu.pl 